\title{
ENCLOSURES AND SEMI-ANALYTIC DISCRETIZATION OF BOUNDARY VALUE PROBLEMS
}

\author{
C. GROSSMANN \\ Department of Mathematics, Kuwait University \\ P.O. Box 5969, Safat 13060, Kuwait \\ E-mail: GROSS@MATH-1.SCI.KUNIV.EDU.KW
}

1. Introduction. Nonlinear boundary value problems as a rule require numerical methods for finding an approximate solution. In some applications not only a numerical solution is wanted, but also a uniform enclosure of the solution of the original problem. The monotone discretization technique developed and investigated in the last years (compare e.g. [9], [23], [27], [28]) forms a powerful tool for the generation of two-sided enclosures of two-point boundary value problems. This method rests on:

- a simplification of the occurring operators,

- a piecewise analytic solution of the generated auxiliary problems,

- monotonicity properties of certain linear parts of the differential operator.

Moreover, the principle of piecewise simplification of the operators occurring in the given boundary value problems is an efficient discretization technique which takes into account the specific behaviour of the boundary value problem to a greater extent than a standard discretization which bases on Taylor expansions only. This makes the proposed technique more adapted to weakly singular or to singularly perturbed problems (see e.g. [31], [42]). Additionally, if the semianalytic discretization is used to generate two-sided enclosures the bounds obtained can be applied to establish a local grid refinement strategy (see [10], [28]).

The aim of the present paper consists in giving a survey of monotone discretization techniques and their application to different types of problems. Because the main principles of monotone discretization have been summarized in [43]

1991 Mathematics Subject Classification: 35B15, 41A65, 65J10, 65L10, 81G45.

This research was partially supported by Kuwait University grant SM-070.

The paper is in final form and no version of it will be published elsewhere. 
here we concentrate our attention on more recent developments. As special applications of monotone discretization we report on the results of the papers [25] and [31] in Section 5.

Let $V, W$ denote some semi-ordered Banach spaces. For operators $L, F: V \rightarrow$ $W$ we consider the equation

$$
L y=F y
$$

as an abstract reference problem. The weakly nonlinear two-point boundary value problem

$$
\begin{aligned}
-y^{\prime \prime}(x) & =f(x, y(x)), \quad x \in \Omega:=(0,1), \\
y(0) & =y(1)=0
\end{aligned}
$$

with some smooth function $f(\cdot, \cdot)$ can be considered as a simple model problem for $(1)$. In this case we choose the Sobolev spaces

$$
V=H_{0}^{1}(\Omega), \quad W=H^{-1}(\Omega)
$$

as $V$ and $W$, respectively. Furthermore, the operators $L, F: H_{0}^{1}(\Omega) \rightarrow H^{-1}(\Omega)$ are defined by

$$
\langle L u, v\rangle=\int_{\Omega} u^{\prime}(x) v^{\prime}(x) d x, \quad\langle F u, v\rangle=\int_{\Omega} f(x, u(x)) v(x) d x
$$

for any $u, v \in V$. Then (1) is just the usual weak formulation of the boundary value problem (2).

2. A semi-analytic discretization technique. First, we sketch the method proposed in [21] for linear problems. Later, the described idea will be applied to nonlinear problems by means of Picard iterations or by successive linearizations. Let us investigate the operator equation

$$
L y=f
$$

with some linear continuous mapping $L: V \rightarrow W$ and some given $f \in W$. We assume a constant $\gamma>0$ to exist such that the following stability condition holds:

$$
\|L u\| \geq \gamma\|u\| \quad \text { for any } u \in V .
$$

Here and in the sequel $\|\cdot\|$ denotes the norm in $V$ as well as in $W$. Let $L_{h}$ and $f_{h}$ denote some approximations of $L$ and $f$, respectively, converging in the following sense:

$$
\lim _{h \rightarrow+0}\left\|L_{h}-L\right\|=0, \quad \lim _{h \rightarrow+0}\left\|f_{h}-f\right\|=0 .
$$

Now, we replace the original problem (4) by auxiliary problems

$$
L_{h} y_{h}=f_{h} \text {. }
$$

The stability and convergence of the solutions $y_{h}$ of the problems (7) to the solution $y$ of (4) are stated in 
LEMmA 1. There exists some $h_{0}>0$ such that the equations (7) have a unique solution $y_{h}$ for any $h \in\left(0, h_{0}\right]$. Furthermore, the estimate

$$
\left\|y-y_{h}\right\| \leq c_{1}\left\|f-f_{h}\right\|+c_{2}\|f\|\left\|L-L_{h}\right\| \quad \text { for any } h \in\left(0, h_{0}\right]
$$

holds with some $c_{1}, c_{2}>0$ independent of $h$ and $f$.

Proof. The assumption (5) guarantees $\left\|L^{-1}\right\| \leq 1 / \gamma$. Using the approximation property (6) and perturbation theory, we know $L_{h}$ to be invertible for sufficiently small $h>0$. In fact, (5) leads directly to

$$
\left\|L_{h} u\right\| \geq\left(\gamma-\left\|L_{h}-L\right\|\right)\|u\| \quad \text { for any } u \in V .
$$

Due to (6), some $c, h_{0}>0$ exist such that

$$
\left\|L_{h} u\right\| \geq c\|u\| \quad \text { for any } u \in V, h \in\left(0, h_{0}\right] .
$$

This results in $\left\|L^{-1}\right\| \leq 1 / c$ for any $h \in\left(0, h_{0}\right]$. From (4) and (7) we obtain

$$
L_{h}\left(y_{h}-y\right)+\left(L_{h}-L\right) y=f_{h}-f .
$$

Thus we have

$$
\left\|y_{h}-y\right\| \leq \frac{1}{c}\left(\left\|f_{h}-f\right\|+\frac{1}{\gamma}\|f\|\left\|L_{h}-L\right\|\right) \quad \text { for any } h \in\left(0, h_{0}\right] .
$$

In [21] we considered different applications of this lemma. A simple realization of the principle of approximate operators is given by a piecewise simplification of the coefficients of the differential operator $L$ and of the right hand side $f$.

Let $\left\{x_{i}\right\}_{i=0}^{N}$ be a grid on the interval $\bar{\Omega}$, i.e.

$$
0=x_{0}<x_{1}<\ldots<x_{N-1}<x_{N}=1 .
$$

We set $h_{i}=x_{i}-x_{i-1}, \Omega_{i}=\left(x_{i-1}, x_{i}\right), i=1(1) N$ and $h=\max _{i} h_{i}$, which defines the step size of the grid. We assume that the grid is quasi-uniform, i.e. $h_{i} \geq c h$, $i=1(1) N$. Here and in the sequel $c, c_{1}, c_{2}, \ldots$ denote some positive constants which can be different at different occurrences. Let

$$
P_{m}:=\left\{v:\left.v\right|_{\Omega_{i}} \text { a polynomial of degree } \leq m\right\}
$$

be the space of piecewise polynomials of maximal degree $m$. We consider the differential operator $L$ defined by

$$
\langle L u, v\rangle=\int_{\Omega}\left(u^{\prime} v^{\prime}+s u^{\prime} v+t u v\right) d x \quad \text { for any } u, v \in V
$$

with (piecewise) sufficiently smooth functions $s(\cdot), t(\cdot)$. An approximate operator $L_{h}$ can be given by

$$
\left\langle L_{h} u, v\right\rangle=\int_{\Omega}\left(u^{\prime} v^{\prime}+s_{h} u^{\prime} v+t_{h} u v\right) d x \quad \text { for any } u, v \in V
$$

with some $s_{h}, t_{h} \in P_{m}$. From the definition of the operators $L$ and $L_{h}$ we obtain the estimate

$$
\left\|L_{h}-L\right\| \leq\left\|s_{h}-s\right\|_{+\infty}+\left\|t_{h}-t\right\|_{+\infty} .
$$


If the originally given operator $L$ is stable in the sense of (5) we can apply Lemma 1 to this approximation. Let us select $s_{h}, t_{h} \in P_{0}$ and $f_{h} \in P_{m}$; then the solution of the auxiliary problem (7) can be represented by standard functions explicitly. The solution $y_{h}$ forms an adapted exponentially fitted spline (compare [23], [42]). In the case $s_{h}, t_{h} \notin P_{0}$ some difficulties arise because the exact solution of (7) is not, as a rule, analytically available. However, this restricts the order of approximation of the scheme (7) for problem (4). One can overcome this obstacle by combining (7) with an iteration

$$
L_{h} y_{h}^{k+1}=f_{h}-\left(L-L_{h}\right) y_{h}^{k}, \quad k=0,1, \ldots
$$

In connection with nonlinear problems and successive linearizations this technique has been investigated e.g. in [24].

Let us consider the general problem (1) with a selfadjoint operator $L$ related to the following two-point boundary value problem:

$$
-\left(a(x) y^{\prime}\right)^{\prime}(x)=f(x, y(x)) \quad \text { in } \Omega, \quad y(0)=y(1)=0 .
$$

The operator $F$ is given by (3). Then an approximate linearization $D_{h}(y)$ of $F$ at $y$ on the given grid $\left\{x_{i}\right\}_{i=0}^{N}$ can be defined by

$$
\left\langle D_{h}(y) z, v\right\rangle=\int_{\Omega} d_{h}[y](x) z(x) v(x) d x \quad \text { for any } z, v \in H_{0}^{1}(\Omega),
$$

with some function $d_{h}[y] \in P_{0}$ depending on the actual iterate $y$ as follows:

$$
d_{h}[y]=\frac{1}{2}\left[\frac{\partial f}{\partial y}\left(x_{i-1}, y\left(x_{i-1}\right)\right)+\frac{\partial f}{\partial y}\left(x_{i}, y\left(x_{i}\right)\right)\right], \quad x \in \Omega_{i}, i=1(1) N .
$$

As a simplification of the operator $L$ we take

$$
\left\langle L_{h} y, v\right\rangle=\int_{\Omega} a_{h}(x) y^{\prime}(x) v^{\prime}(x) d x \quad \text { for any } y, v \in V
$$

with some $a_{h} \in P_{0}$. A realization of (12) adapted to the nonlinear problem (13) is now given by

$$
\left(L_{h}+D_{h}\left(y^{k}\right)\right) y^{k+1}=\left(F+D_{h}\left(y^{k}\right)\right) y^{k}+\left(L_{h}-L\right) y^{k}, \quad k=0,1, \ldots,
$$

with some initial guess $y^{0} \in V$. Assuming $\lim _{h \rightarrow+0}\left\|a-a_{h}\right\|_{h}=0$, (17) actually forms an approximate version of Newton's method. By standard arguments for proving the convergence of perturbed Newton techniques (compare, e.g., [37]) this results in

THEOREM 1. Let $y \in V$ denote some solution of problem (13) that is stable in the sense that

$$
\left\langle\left(L-F^{\prime}(y)\right) u, u\right\rangle=\gamma\|u\|^{2} \quad \text { for any } u \in V
$$

with some $\gamma>0 ; f(\cdot, \cdot)$ is supposed to be Lipschitz-continuously differentiable. Then some $\bar{h}>0$ and $\delta>0$ exist such that method (17) for any $h \in(0, \bar{h}]$ 
and $\left\|y^{0}-y\right\| \leq \delta$ generates a well-defined sequence $\left\{y^{k}\right\}$ converging to $y$. More precisely, we have some constants $c_{1}, c_{2}>0$ such that

$$
\left\|y^{k+1}-y\right\| \leq\left(c_{1}\left\|y^{k}-u\right\|+c_{2} h\right)\left\|y^{k}-y\right\|, \quad k=0,1, \ldots
$$

Remarks. 1. The inequalities (19) show the method (17) to be asymptotically superlinearly convergent for $h \rightarrow+0$.

2. The method (17) is not implementable in the strict sense because it cannot, as a rule, be realized using available finite-dimensional spaces with dimensions bounded from above. Because of this, below we modify the method (17) to make it implementable.

Let $C_{h}(\bar{\Omega})$ denote the space of piecewise continuous functions with jumps at the grid points only. We introduce a projection $\pi_{h}: C_{h}(\bar{\Omega}) \rightarrow P_{1}$ by taking

$$
\left[\pi_{h} w\right](x)=w\left(x_{i-1}+0\right) \frac{x_{i}-x}{h_{i}}+w\left(x_{i}-0\right) \frac{x-x_{i-1}}{h_{i}}, \quad x \in \Omega_{i}, i=1(1) N .
$$

Furthermore, in view of the smoothing properties of $L_{h}^{-1}$ we can restrict our investigations to the space $U:=V \cap C_{h}^{2}(\bar{\Omega})$ (compare [24]) and we split the operator $L-L_{h}$ as

$$
\left(L-L_{h}\right) y=\left(Q_{h}+R_{h}\right) y \quad \text { for any } y \in U,
$$

with $Q_{h}, R_{h}: U \rightarrow W$ defined by

$$
\begin{aligned}
& \left\langle Q_{h} y, v\right\rangle=-\sum_{i=1}^{N} \int_{\Omega_{i}}\left(\left(a_{h}-a\right) y^{\prime}\right)^{\prime}(x) v(x) d x \quad \text { for any } y \in U, v \in V, \\
& \left\langle R_{h} y, v\right\rangle=\sum_{i=1}^{N-1}\left\{\left[\left(a_{h}-a\right) y^{\prime}\right]\left(x_{i}-0\right)-\left[\left(a_{h}-a\right) y^{\prime}\right]\left(x_{i}+0\right)\right\} v\left(x_{i}\right)
\end{aligned}
$$

for any $y \in U, v \in V$

Via integration by parts we obtain the identity in $W$

$$
\left(L-L_{h}\right) y=\left(Q_{h}+R_{h}\right) y \quad \text { for any } y \in U .
$$

In [24] we proposed the method

$$
\begin{aligned}
\left(L_{h}+D_{h}\left(y_{h}^{k}\right)\right) & y_{h}^{k+1} \\
& =\pi_{h}\left(F+D_{h}\left(y_{h}^{k}\right)\right) y_{h}^{k}+\pi_{h} Q_{h} y_{h}^{k}+R_{h} y_{h}^{k}, \quad k=0,1, \ldots,
\end{aligned}
$$

with some initial function $y^{0} \in U$. Unlike (17) this method can be realized using known finite-dimensional representations by piecewise exponential splines (compare, e.g., [22], [42]). However, we have to make the following additional assumption on the variation of the function $a(\cdot)$ :

$$
\max _{1 \leq i \leq N} \frac{\left\|a^{\prime}\right\|_{C\left(\bar{\Omega}_{i}\right)}}{a_{i}} \leq \beta<1
$$

with some $\beta \in(0,1)$. Now we can prove (see [24]) the following convergence result: 
THEOREM 2. Let $y \in V$ denote some solution of problem (13) which satisfies the regularity assumption (18). Furthermore, $y$ is supposed to be twice continuously differentiable. Then there exist $\delta>0$ and $\bar{h}>0$ such that the method (25) is well defined for any $h \in(0, \bar{h}]$ and $y_{h}^{0} \in V \cap C^{2}(\bar{\Omega})$ with $\left\|y_{h}^{0}-y\right\|_{h, 2}<\delta$. The sequence $\left\{y_{h}^{k}\right\}$ generated by the method converges to some $y_{h}$ approximating the solution $y$ of (13) with the accuracy

$$
\left\|y_{h}-y\right\| \leq c h^{2}
$$

with some $c>0$.

A completely different technique, the method of truncated local Taylor expansions considered in [1], can be investigated with the perturbed operator approach as well. In [21] we analyzed this method by relating the obtained auxiliary problems to a specific perturbation of the original one.

Adapted discretization schemes for singularly perturbed problems using perturbed operators have been investigated in [31], [40], [42]. By means of Airy functions in [42] a technique similar to (11) with $s_{h}, t_{h} \in P_{1}$ has been proposed. It should be mentioned that an important property of the linear operator $L$, namely some weak maximum principle, was used to prove that the schemes are uniformly convergent with respect to the singular perturbation parameter (compare Subsection 5.3).

As already remarked, the perturbed operator technique is especially efficient if some singularities occur which result in difficulties in standard discretization methods. The proposed principle in combination with directional projections in [25] is applied to generate enclosures of the solution of the Thomas-Fermi equation. We describe this method briefly in Section 5 of this paper.

3. Generation of enclosures by monotone discretization. Let us consider the abstract problem (1), i.e.

$$
L y=F y .
$$

Additionally we suppose the operator $L-F$ to be of monotone kind in the semiordering of the spaces $V$ and $W$. This means that

$$
(L-F) u \leq(L-F) v \Rightarrow u \leq v
$$

With the natural a.e. pointwise semi-ordering of $V=H_{0}^{1}(\Omega)$ and with the related dual ordering in $W=H^{-1}(\Omega)$ the weak maximum principle and the monotonicity assumption

$$
f(x, s) \geq f(x, t) \quad \text { for any } x \in \bar{\Omega}, s \leq t
$$

guarantee $(28)$ for the related operator $L-F$.

Let $W_{h} \subset W$ denote some finite-dimensional subspace. In most of the realizations (compare [23], [29]) we selected $W_{h}=P_{m}$ on a grid $\left\{x_{i}\right\}_{i=0}^{N}$ over the interval $\bar{\Omega}$. Mappings $\underline{\pi}_{h}, \bar{\pi}_{h}: W \rightarrow W_{h}$ are called lower and upper bounding operators, 
respectively, if

$$
\underline{\pi}_{h} w \leq w \leq \bar{\pi}_{h} w \quad \text { for any } w \in W .
$$

Using the smoothing properties of $L^{-1}$ the domain of $\underline{\pi}_{h}, \bar{\pi}_{h}$ can be additionally restricted, e.g. to $C_{h}(\bar{\Omega})$, in most of the applications.

By means of $\underline{\pi}_{h}, \bar{\pi}_{h}$ we modify the original problem (27) to the following auxiliary problems:

$$
\underline{y}_{h}, \bar{y}_{h} \in V: \quad L \underline{y}_{h}=\underline{\pi}_{h} F \underline{y}_{h}, \quad L \bar{y}_{h}=\bar{\pi}_{h} F \bar{y}_{h} .
$$

As a direct consequence of the properties (28), (30) we obtain

THEOREM 3. Let $y$ and $\underline{y}_{h}, \bar{y}_{h}$ solve (27) and the related problems (31), respectively. Then the following enclosure holds:

$$
\underline{y}_{h} \leq y \leq \bar{y}_{h} .
$$

Proof. Equations (31) with property (30) result in

$$
\begin{aligned}
(L-F) \underline{y}_{h} & \leq L \underline{y}_{h}-\underline{\pi}_{h} F \underline{y}_{h}=0=(L-F) y \\
& =L \bar{y}_{h}-\bar{\pi}_{h} F \bar{y}_{h} \leq(L-F) \bar{y}_{h} .
\end{aligned}
$$

Since $L-F$ is of monotone kind this yields (32).

Remarks. 1. The assumption (28) is quite restrictive. It can be relaxed by additionally considering monotonically convergent iteration techniques (compare Section 4).

2. The simplest bounding mappings are given by $\underline{\pi}_{h}=\underline{p}_{h}, \bar{\pi}_{h}=\bar{p}_{h}$ with $\underline{p}_{h}, \bar{p}_{h}: C_{h}(\bar{\Omega}) \rightarrow P_{0}$ defined by

$$
\left[\underline{p}_{h}\right](x)=\inf _{\xi \in \Omega_{i}} w(\xi), \quad\left[\bar{p}_{h}\right](x)=\sup _{\xi \in \Omega_{i}} w(\xi) \quad \text { for } x \in \Omega_{i}, i=1(1) N .
$$

The rate of convergence of monotone discretization in dependence on the mesh size $h>0$ is mainly influenced by the approximation properties of the bounding operators. Because of the piecewise constant replacement, the method (31) with $\pi_{h}=p_{h}$ gives $(33)$ coming from

$$
\left\|y_{h}-y\right\|=O(h)
$$

only (compare [27]). Here and in the sequel we omit the indication whether we deal with the lower or with the upper bounding operator if the related result holds for both of them. Higher order approximations are obtained by a shifted polynomial interpolation (see [29], [30]). If $S_{h}: C_{h}(\bar{\Omega}) \rightarrow C_{h}(\bar{\Omega})$ denotes a piecewise approximating operator then a bounding operator can be defined by

$$
\pi_{h} w:=\left[S_{h}+p_{h}\left(I-S_{h}\right)\right] w
$$

with $p_{h}$ given by (33). The bounding property (30) immediately results from the bounding property of $p_{h}$ and (34). Indeed, we have

$$
\bar{\pi}_{h} w=\left(S_{h}+\bar{p}_{h}\left(I-S_{h}\right)\right) w \geq\left(S_{h}+\left(I-S_{h}\right)\right) w=w .
$$


The approximation behaviour of $\pi_{h}$ is obtained from

$$
\left(I-\pi_{h}\right) w=\left(I-p_{h}\right)\left(I-S_{h}\right) w .
$$

Since $\left\|\left(I-p_{h}\right) w\right\|_{+\infty} \leq 2\|w\|_{+\infty}$ the order of approximation of $\pi_{h}$ defined by (34) mainly depends on the approximation properties of the operator $S_{h}$. For further details we refer to [29], [30].

In Theorem 3 the existence of the solution $y$ of (27) as well as the existence of the solutions $y_{h}, \bar{y}_{h}$ of the auxiliary problems have been supposed. Under mild additional conditions we investigated this problem in [27]. Another approach is via monotone iteration which we now describe.

4. Monotone iteration discretization. The monotone discretization technique as considered in Section 3 suffers from some serious drawbacks:

- The generated auxiliary problems (31) are highly nonlinear because of the behaviour of the bounding operators $\pi_{h}$. Thus, an adapted iteration method is required for solving (31) efficiently.

- The assumption (28), i.e. that $L-F$ is of monotone kind, restricts the class of problems which can be handled with monotone discretization.

A possible way to relax the assumptions needed for monotone discretization methods is to combine the technique with a monotone iteration scheme. First we sketch the basic principle of monotone iterations. Let $T: V \rightarrow V$ be some operator and $\underline{u}^{0}, \bar{u}^{0} \in V$ some elements such that

$$
\begin{gathered}
\underline{u}^{0} \leq \bar{u}^{0}, \\
\underline{u}^{0} \leq T \underline{u}^{0}, \quad \bar{u}^{0} \geq T \bar{u}^{0}, \\
\underline{u}^{0} \leq u \leq v \leq \bar{u}^{0} \Rightarrow T u \leq T v .
\end{gathered}
$$

These properties lead to

LEMMA 2. Under the above assumptions the sequences $\left\{\underline{u}^{k}\right\},\left\{\bar{u}^{k}\right\}$ generated by the iterations

$$
\underline{u}^{k+1}=T \underline{u}^{k}, \quad \bar{u}^{k+1}=T \bar{u}^{k}, \quad k=0,1, \ldots,
$$

satisfy

$$
\underline{u}^{0} \leq \underline{u}^{1} \leq \ldots \leq \underline{u}^{k-1} \leq \underline{u}^{k} \leq \ldots \leq \bar{u}^{k} \leq \bar{u}^{k-1} \leq \ldots \leq \bar{u}^{1} \leq \bar{u}^{0} .
$$

If, additionally, the operator $T$ has some fixed point property (compare, e.g., [45]) then at least one $y \in\left[\underline{u}^{k}, \bar{u}^{k}\right]$ exists which is a fixed point of $T$, i.e. $y=T y$.

The fixed point property in our cases of two-point boundary value problems can be proved by compact embeddings as guaranteed by the Rellich-Kondrashov theorem. 
To apply monotone iterations to auxiliary problems (31) some modifications are needed. Let us consider problem (27) and let $\underline{y}^{0}, \bar{y}^{0} \in V$ be given such that

$$
\underline{y}^{0} \leq \bar{y}^{0} \text {. }
$$

Furthermore, we assume that some constant $\varrho>0$ exists which guarantees

$$
\underline{y}^{0} \leq u \leq v \leq \bar{y}^{0} \Rightarrow(F+\varrho I) u \leq(F+\varrho I) v .
$$

For the bounding operators $\underline{\pi}_{h}, \bar{\pi}_{h}$, additionally, the starting inequalities

$$
(L+\varrho I) \underline{y}^{0} \leq \underline{\pi}_{h}(F+\varrho I) \underline{y}^{0}, \quad(L+\varrho I) \bar{y}^{0} \geq \bar{\pi}_{h}(F+\varrho I) \bar{y}^{0}
$$

should be satisfied. Now we have the following basic algorithm:

$$
\begin{array}{r}
(L+\varrho I) \underline{y}^{k+1}=\underline{\pi}_{h}(F+\varrho I) \underline{y}^{k}, \quad(L+\varrho I) \bar{y}^{k+1}=\bar{\pi}_{h}(F+\varrho I) \bar{y}^{k}, \\
k=0,1, \ldots
\end{array}
$$

THEOREM 4. Let $L+\varrho I$ be an operator of monotone kind and let $(L+\varrho I)^{-1}$ have the smoothing property $(L+\varrho I)^{-1} f \in H^{2}(\Omega)$ for any $f \in P_{0}$. Then the algorithm (38)-(41) generates sequences $\left\{y^{k}\right\},\left\{\bar{y}^{k}\right\}$ with

$$
\underline{y}^{0} \leq \underline{y}^{1} \leq \ldots \leq \underline{y}^{k-1} \leq \underline{y}^{k} \leq \ldots \leq \bar{y}^{k} \leq \bar{y}^{k-1} \leq \ldots \leq \bar{y}^{1} \leq \bar{y}^{0} .
$$

Furthermore, each of the intervals $\left[\underline{y}^{k}, \bar{y}^{k}\right]$ contains at least one solution $y \in V$ of the original problem (27).

Remarks. 1. The iteration process (41) can be accelerated by using some modified linearization of $F$ instead of the operator $\varrho I$. This results in an iteration technique

$$
\begin{array}{r}
\left(L+D_{k}\right) \underline{y}^{k+1}=\underline{\pi}_{h}\left(F+D_{k}\right) \underline{y}^{k}, \quad\left(L+D_{k}\right) \bar{y}^{k+1}=\bar{\pi}_{h}\left(F+D_{k}\right) \bar{y}^{k}, \\
k=0,1, \ldots,
\end{array}
$$

with $\left[D_{k} y\right](x):=d_{k}(x) y(x)$ and some adapted $d_{k} \in P_{0}$. To prove the monotonicity of $\left\{y^{k}\right\},\left\{\bar{y}^{k}\right\}$ additional investigations are necessary if $D_{k}$ is changed during the iteration (compare [9]).

2. In practical implementations the bounding operators $\underline{p}_{h}, \bar{p}_{h}$ are replaced by available piecewise constant bounds. In this case the bounds are, as a rule, not monotone. This can be overcome by additional intersections of the bounding intervals as known from interval analysis (see [9]).

In [8] we give an interval realization of the monotone iteration discretization (MID) method. The needed interval operations can be efficiently implemented in advanced programming languages like, e.g., PASCAL-SC [34].

5. Application of monotone discretizations to selected problems. In this section we mainly report on the results of [25], [31].

5.1. The Thomas-Fermi equation. In this subsection we deal with the original Thomas-Fermi equation

$$
y^{\prime \prime}=x^{-1 / 2} y^{3 / 2}
$$


with the boundary condition

$$
y(0)=1, \quad y(a)=0 .
$$

Here $a>0$ denotes some given constant. The boundary value problem (43), (44) occurs in the investigation of potentials and charge densities of ionized atoms. Existence, uniqueness and smoothness properties of the solution of $(43),(44)$ have been shown in various publications (see, e.g., [16]). Let $\Omega:=(0, a)$. In $H_{0}^{1}(\Omega)$ we use the same norm as in $H^{1}(\Omega)$. For $V:=H^{1}(\Omega)$ and $W:=H^{-1}(\Omega)$ we define operators $L, F: V \rightarrow W$ by

$$
\begin{aligned}
& \langle L u, v\rangle:=\int_{\Omega} u^{\prime}(x) v^{\prime}(x) d x \quad \text { for any } u \in V, v \in H_{0}^{1}(\Omega), \\
& \langle F u, v\rangle:=-\int_{\Omega} x^{-1 / 2}[u(x)]_{+}^{3 / 2} v(x) d x \quad \text { for any } u \in V, v \in H_{0}^{1}(\Omega) .
\end{aligned}
$$

Here $[\cdot]_{+}$denotes the positive part, i.e. $[t]_{+}:=\max \{0, t\}$ for any $t \in \mathbb{R}$. The operator $L-F$ is coercive on the subspace of $V$ realizing the boundary conditions (44), i.e. for

$$
U:=\{v \in V: v(0)=1, v(a)=0\}
$$

we have

$$
\langle(L-F) u-(L-F) v, u-v\rangle \geq \gamma\|u-v\|^{2} \quad \text { for any } u, v \in U
$$

with some constant $\gamma>0$. Furthermore, $L-F$ is of monotone kind. Now, we define a finite-dimensional subspace $W_{h} \subset W$ by

$$
W_{h}:=\operatorname{lin}\left\{\zeta_{i j}\right\}_{i=1, j=0}^{N}
$$

with

$$
\zeta_{i j}(x):= \begin{cases}x^{-1 / 2}\left(x_{i}-x\right)^{j}, & x \in \Omega_{i} \\ 0 & \text { otherwise }\end{cases}
$$

$i=1(1) N, j=0,1$. Thus, the functions $v \in W_{h}$ can be represented by

$$
v(x)=\sum_{i=1}^{N} \sum_{j=0}^{1} w_{i j} \zeta_{i j}(x) \quad \text { a.e. in } \Omega
$$

with some $w_{i j} \in \mathbb{R}, i=1(1) N, j=0,1$.

In monotone discretization as shown in Section 3 we replace the nonlinear operator $F: V \rightarrow W$ by appropriate bounding operators $\underline{F}_{h}, \bar{F}_{h}: U \subset V \rightarrow$ $W_{h} \subset W$, respectively. Then the auxiliary problems

$$
\underline{y}_{h}, \bar{y}_{h} \in U: \quad\left(L-\underline{F}_{h}\right) \underline{y}_{h}=0, \quad\left(L-\bar{F}_{h}\right) \bar{y}_{h}=0
$$

are to be solved instead of (43), (44). We define

$$
\left[\underline{F}_{h} u\right](x):=-x^{-1 / 2}\left(\left[u_{i}\right]_{+}^{3 / 2}-\frac{\left[u_{i}\right]_{+}^{3 / 2}-\left[u_{i-1}\right]_{+}^{3 / 2}}{h_{i}}\left(x_{i}-x\right)\right),
$$




$$
\left[\bar{F}_{h} u\right](x):=-x^{-1 / 2}\left(\left[u_{i}\right]_{+}^{3 / 2}-\frac{3}{2}\left[u_{i}\right]_{+}^{1 / 2}\left[u_{i}^{\prime}\right]_{-}\left(x_{i}-x\right)\right),
$$

for any $x \in \Omega_{i}$.

Here $[t]_{-}:=\min \{0, t\}, t \in \mathbb{R}$, and $u_{i}:=u\left(x_{i}\right), u_{i}^{\prime}:=u^{\prime}\left(x_{i}\right), i=1(1) N$. Bounding operators of the form (47), (48) replacing the original functions by piecewise secants and tangents, respectively, have been proposed in [48] and applied in [17], for example.

Now we take advantage of the linearity of the operator $L$ and its relation to local boundary value problems. We define functions $\phi_{i}, \psi_{i j}$ by

$$
\phi_{i}(x)= \begin{cases}\left(x-x_{i-1}\right) / h_{i}, & x \in \Omega_{i} \\ \left(x_{i+1}-x\right) / h_{i+1}, & x \in \Omega_{i+1} \\ 0 & \text { otherwise }\end{cases}
$$

with $i=0(1) N$

$$
\psi_{i 0}(x)= \begin{cases}\frac{4}{3}\left(x^{3 / 2}-x_{i-1}^{3 / 2} \phi_{i-1}(x)-x_{i}^{3 / 2} \phi_{i}(x)\right), & x \in \Omega_{i}, \\ 0 & \text { otherwise }\end{cases}
$$

and

$$
\psi_{i 1}(x)= \begin{cases}x_{i} \psi_{i 0}-\frac{4}{15}\left(x^{5 / 2}-x_{i-1}^{5 / 2} \phi_{i-1}(x)-x_{i}^{5 / 2} \phi_{i}(x)\right), & x \in \Omega_{i}, \\ 0 & \text { otherwise }\end{cases}
$$

with $i=1(1) N$

From these definitions we obtain

$$
-\phi_{i}^{\prime \prime}(x)=0 \text { a.e. in } \Omega, \quad \phi_{i}\left(x_{k}\right)=\delta_{i k}
$$

and

$$
-\psi_{i j}^{\prime \prime}(x)+\zeta_{i j}(x)=0 \quad \text { a.e. in } \Omega, \quad \psi_{i j}\left(x_{k}\right)=0 .
$$

Taking $y_{i} \in \mathbb{R}, i=0(1) N$, as parameters the superposition principle results in the representation

$$
y_{h}(x)=\sum_{i=0}^{N} y_{i} \phi_{i}(x)+\sum_{i=1}^{N} \sum_{j=0}^{1} w_{i j} \psi_{i j}(x)
$$

of the solutions $y_{h}$ of the auxiliary problems (46). Here the $w_{i j}$ denote the coefficients of $F_{h} y_{h}$ with respect to the base $\left\{\zeta_{i j}\right\}$ of $W_{h}$ and $y_{0}=1, y_{N}=0$ are given by the boundary conditions (44). We obtain the remaining coefficients $y_{i}$, $i=1(1) N-1$, in (51) from the differentiability of $y_{h}$ at the inner grid points. Taking the supports of $\phi_{i}, \psi_{i j}$ into account this leads to

$$
\frac{u_{i}-u_{i-1}}{h_{i}}+\sum_{j=0}^{1} w_{i j} \psi_{i j}^{\prime}\left(x_{i}-0\right)=\frac{u_{i+1}-u_{i}}{h_{i+1}}+\sum_{j=0}^{1} w_{i+1 j} \psi_{i+1 j}^{\prime}\left(x_{i}+0\right),
$$

with $i=1(1) N-1$.

The existence and local uniqueness of solutions $y_{h}, \bar{y}_{h}$ of the auxiliary problems (46) for sufficiently small $h>0$ can be shown by the technique used in [27]. 
THEOREM 5. Let $\underline{y}_{h}, \bar{y}_{h} \in W$ denote solutions of the auxiliary problems (46). Then the estimates

$$
\underline{y}_{h} \leq y \leq \bar{y}_{h}
$$

hold for the solution $y$ of the original problem (43), (44).

Pr o of. The definitions (47), (48) of the bounding operators $\underline{F}_{h}, \bar{F}_{h}$ give

$$
\underline{F}_{h} u \leq 0, \quad \bar{F}_{h} u \leq 0 \quad \text { for any } u \in W .
$$

Together with (46) and the definition of the operator $L$ this leads to the convexity of the functions $\underline{y}_{h}, \bar{y}_{h}$. Because the functions $[\cdot]_{+}$and $(\cdot)^{3 / 2}$ are convex and nondecreasing, also the superpositions $\left[\underline{y}_{h}\right]_{+}^{3 / 2},\left[\bar{y}_{h}\right]_{+}^{3 / 2}$ are convex. Now, using (47) we obtain

$$
\underline{F}_{h} \underline{y}_{h} \leq F \underline{y}_{h} .
$$

Because of $\left[\bar{y}_{h}\right]_{+}^{3 / 2} \geq 0$ and $\left[\bar{y}_{h}\right]_{+}^{3 / 2}(a)=0$ we have

$$
\left(\left[\bar{y}_{h}\right]_{+}^{3 / 2}\right)^{\prime}(a) \leq 0 .
$$

With the convexity of $\left[\bar{y}_{h}\right]_{+}^{3 / 2}$ this leads to

$$
\left(\left[\bar{y}_{h}\right]_{+}^{3 / 2}\right)^{\prime} \leq 0 \quad \text { on } \Omega \text {. }
$$

Thus, the identity

$$
\left[\bar{F}_{h} \bar{y}_{h}\right](x)=-x^{-1 / 2}\left(\left[\bar{y}_{i}\right]_{+}^{3 / 2}-\frac{3}{2}\left[\bar{y}_{i}\right]_{+}^{1 / 2}\left[\bar{y}_{i}^{\prime}\right]_{-}\left(x_{i}-x\right)\right), \quad x \in \Omega_{i},
$$

holds with $\bar{y}_{i}=\bar{y}_{h}\left(x_{i}\right), \bar{y}_{i}^{\prime}=\bar{y}_{h}^{\prime}\left(x_{i}\right)$. Because of the known structure of the solution $\bar{y}_{h}$ its first derivative $\bar{y}_{h}^{\prime}$ is explicitly available and need not be approximated numerically.

Using the convexity of $\left[\bar{y}_{h}\right]_{+}^{3 / 2}$, we now obtain

$$
F \bar{y}_{h} \leq \bar{F}_{h} \bar{y}_{h} .
$$

By the properties of the bounding operators this leads to

$$
(L-F) \underline{y}_{h} \leq\left(L-\underline{F}_{h}\right) \underline{y}_{h}=0=(L-F) y=\left(L-\bar{F}_{h}\right) \bar{y}_{h} \leq(L-F) \bar{y}_{h} .
$$

Because the operator $L-F$ is of monotone kind this completes the proof.

R e m a r k. Basing on the monotonicity of $\underline{y}_{h}, \bar{y}_{h}$ shown in the proof above one can take

$$
\left[\underline{F}_{h} u\right](x):=-x^{-1 / 2}\left[u_{i-1}\right]_{+}^{3 / 2}, \quad x \in \Omega_{i},
$$

$$
\left[\bar{F}_{h} u\right](x):=-x^{-1 / 2}\left[u_{i}\right]_{+}^{3 / 2}, \quad x \in \Omega_{i},
$$

as simple bounding operators approximating $F$ with order $O(h)$.

Next, we characterize the order of approximation of the solution $y$ of (43), (44) by $\underline{y}_{h}, \bar{y}_{h}$. 
TheOREM 6. There exists some $c>0$ such that

$$
\left\|\bar{y}_{h}-\underline{y}_{h}\right\| \leq c h^{2}
$$

for sufficiently small $h>0$.

Proof. From (46) we obtain

$$
0=\left(L-\underline{F}_{h}\right) \underline{y}_{h}=(L-F) \underline{y}_{h}-\left(\underline{F}_{h}-F\right) \underline{y}_{h} .
$$

By coercivity of the operator $L-F$ this results in

$$
\left\|y-\underline{y}_{h}\right\| \leq \frac{1}{\gamma}\left\|\left(\underline{F}_{h}-F\right) \underline{y}_{h}\right\| .
$$

The sequence $\left\{\underline{y}_{h}\right\}$ can be shown to be bounded. Using (46), the smoothing property of $L^{-1}$ and the continuous embedding $H^{2}(\Omega) \hookrightarrow C^{1+1 / 2}(\bar{\Omega})$ (compare [3]) we obtain

$$
\left|\underline{y}_{h}^{\prime}(\xi)-\underline{y}_{h}^{\prime}(\eta)\right| \leq c_{1} h^{1 / 2} \quad \text { for any } \xi, \eta \in \Omega_{i}, i=1(1) N,
$$

with some $c_{1}>0$. Taking the definition (47) of $\underline{F}_{h}$ into account and integrating by parts this leads to

$$
\left|\left\langle\left(\underline{F}_{h}-F\right) \underline{y}_{h}, v\right\rangle\right| \leq c_{2} h^{2}\|v\| \quad \text { for any } v \in V
$$

with some $c_{2}>0$. Thus, we have

$$
\left\|\left(\underline{F}_{h}-F\right) \underline{y}_{h}\right\| \leq c_{2} h^{2} .
$$

Estimate (56) and the analogous result for the upper solution $\bar{y}_{h}$ prove the statement of the theorem.

We refer to [25] for further details of the proposed discretization technique. Numerical implementations for the case $a=1$ yield the following table:

\begin{tabular}{|c|cc|c|cc|}
\hline$x$ & \multicolumn{2}{|c|}{$\underline{y}_{h}(x)$} & $y(x)$ & \multicolumn{2}{c|}{$\bar{y}_{h}(x)$} \\
\hline & $h=0.1$ & $h=0.025$ & & $h=0.025$ & $h=0.1$ \\
\hline 0.0 & 1.00000 & 1.00000 & 1.00000 & 1.00000 & 1.00000 \\
0.1 & 0.84923 & 0.84946 & 0.84947 & 0.84950 & 0.84979 \\
0.2 & 0.72692 & 0.72721 & 0.72723 & 0.72727 & 0.72766 \\
0.3 & 0.61896 & 0.61927 & 0.61929 & 0.61933 & 0.61977 \\
0.4 & 0.52000 & 0.52039 & 0.52041 & 0.52045 & 0.52090 \\
0.5 & 0.42725 & 0.42753 & 0.42755 & 0.42758 & 0.42802 \\
0.6 & 0.33843 & 0.33867 & 0.33869 & 0.33872 & 0.33912 \\
0.7 & 0.25218 & 0.25239 & 0.25240 & 0.25242 & 0.25277 \\
0.8 & 0.16749 & 0.16764 & 0.16765 & 0.16767 & 0.16794 \\
0.9 & 0.08360 & 0.08368 & 0.08369 & 0.08370 & 0.08387 \\
1.0 & 0.00000 & 0.00000 & 0.00000 & 0.00000 & 0.00000 \\
\hline
\end{tabular}

5.2. A nonlinear eigenvalue problem. Some of the underlying ideas as monotone discretization by bounding operators and using convexity can be applied to other types of problems as well. In the case of generalized Emden-Fowler equations considered e.g. in [19] this approach leads to a sequence of finite-dimensional 
nonlinear eigenvalue problems. The related eigenfunctions are represented by piecewise cubic polynomials. It should be mentioned that unlike in [19] here we concentrate our attention on the construction of an adapted discretization of the eigenvalue problem. The generated finite-dimensional systems can be treated by a technique similar to [23], for example. Here we applied some shooting method to solve the finite-dimensional problems.

Let us consider the generalized Emden-Fowler equation (superlinear case, compare [19])

$$
y^{\prime \prime}(x)+\lambda y^{3}(x)=0 \quad \text { in } \Omega:=(0,1), \quad y(0)=y(1)=0
$$

as an example. Related to this problem we define

$$
\langle F u, v\rangle:=\int_{\Omega}[u(x)]_{+}^{3} v(x) d x \quad \text { for any } u, v \in H_{0}^{1}(\Omega) .
$$

Similarly to $(47),(48)$ we apply piecewise secants and piecewise tangents to define bounding operators. But, unlike in the case of the Thomas-Fermi equation, here the solution $u$ is concave and the superposition $u^{3}$ is neither concave nor convex. Thus, we use the piecewise linearizations to the function $u$ itself. This results in the following bounding operators:

$$
\begin{aligned}
& {\left[\bar{F}_{h} u\right](x):=\left[u_{i}-\frac{u_{i}-u_{i-1}}{h_{i}}\left(x_{i}-x\right)\right]_{+}^{3},} \\
& {\left[\underline{F}_{h} u\right](x):=\left[u_{i}-u_{i}^{\prime}\left(x_{i}-x\right)\right]_{+}^{3},}
\end{aligned}
$$

for any $x \in \Omega_{i}$. Now, the finite-dimensional eigenvalue problem can be given in the abstract form

$$
y_{h} \in H_{0}^{1}(\Omega), \quad L y_{h}=\lambda_{h} F_{h} y_{h} .
$$

This problem can be treated in an adapted finite-dimensional space. Because $F_{h} y$ for any $y \in H_{0}^{1}(\Omega)$ are piecewise cubic polynomials we know the solutions $y_{h}$ of (60) to be polynomials of degree five. Finally, we report on some numerical results obtained with the proposed method for the Emden-Fowler equation. With the scaling $y^{\prime}(0)=1$ as used in [19] we solved these problems for various stepsizes $h>0$ on equidistributed grids by a shooting method. Our results are given in the following table:

\begin{tabular}{|l|c|c|c|}
\hline \multicolumn{1}{|c|}{$h$} & $\lambda_{h}$ according to (59) & $\lambda_{h}$ according to (58) & difference \\
\hline 0.1 & 88.0498 & 97.528966 & $9.36 \mathrm{E}-0$ \\
0.01 & 94.475750 & 94.566103 & $9.04 \mathrm{E}-2$ \\
0.001 & 94.535760 & 94.536652 & $8.92 \mathrm{E}-4$ \\
0.0001 & 94.536350 & 94.536358 & $8.00 \mathrm{E}-6$ \\
\hline
\end{tabular}

The bounds converge quadratically as expected. For an example of another technique for solving eigenvalue problems which uses analytic information by taking proper functions into the ansatz of the eigenfunctions we refer to [49]. 
5.3. Singularly perturbed problems. In [31] we applied the concept of monotone discretization to singular as well as to singularly perturbed problems. Let us consider the following singularly perturbed singular problem:

$$
-\varepsilon^{2} \frac{d}{d x}\left(x^{\alpha} \frac{d u}{d x}\right)+p(x) u=f(x), \quad x \in(0,1), \quad u(0)=u(1)=0
$$

with some parameter $\alpha \in(0,1)$ and a perturbation parameter $\varepsilon(0<\varepsilon \ll 1)$. We assume the functions $p$ and $f$ to be sufficiently smooth and we suppose $p(x) \geq$ $p_{0}>0$ for any $x \in(0,1)$ with some constant $p_{0}$.

The results of Section 2 can be used to deduce that problem (61) for any fixed $\varepsilon \neq 0$ admits a unique solution $u \in H_{0}^{1, \alpha}(\Omega)$. Here $H_{0}^{1, \alpha}(\Omega)$ denotes the related weighted Sobolev space equipped with the norm

$$
\|v\|:=\left(\int_{\Omega} x^{\alpha}\left|v^{\prime}(x)\right|^{2} d x\right)^{1 / 2} \quad \text { for any } v \in H_{0}^{1, \alpha}(\Omega) .
$$

Because of the embedding $H_{0}^{1, \alpha} \Omega \hookrightarrow C(\bar{\Omega})$ this solution is continuous. However, the direct application of this embedding leads to a bound of $\|u\|_{L^{\infty}}$ which tends to $+\infty$ for $\varepsilon \rightarrow 0$. But in the singularly perturbed case it is essential to analyze carefully the dependence of all estimates on the perturbation parameter $\varepsilon$. Here the maximum principle is a useful tool. Taking $v(x):=\|f\|_{L^{\infty}} / p_{0}$ and $v(x):=$ $-\|f\|_{L^{\infty}} / p_{0}$, respectively, we obtain the estimate

$$
\max _{x \in[0,1]}|u| \leq \frac{1}{p_{0}} \max _{x \in[0,1]}|f(x)| .
$$

This gives a bound of $\|u\|_{L^{\infty}}$ uniform with respect to $\varepsilon$.

The reduced solution $u_{0}:=f / p$ in general does not satisfy the boundary conditions of the original problem. Therefore, near $x=0$ and $x=1$ a boundary layer exists. Introducing $\xi_{1}=(1-x) / \varepsilon$ the boundary layer correction near $x=1$ satisfies

$$
-\frac{d^{2} v}{d \xi_{1}^{2}}+p(1) v\left(\xi_{1}\right)=0, \quad v(0)=-u_{0}(1), \quad v(+\infty)=0 .
$$

One can easily see that this problem has an exponentially decreasing solution $v_{1}\left(\xi_{1}\right)$. Setting $\xi_{0}=x / \varepsilon^{2 /(2-\alpha)}$ we obtain the boundary value problem

$$
-\frac{d}{d \xi_{0}}\left(\xi_{0}^{\alpha} \frac{d v}{d \xi_{0}}\right)+p(0) v\left(\xi_{0}\right)=0, \quad v(0)=-u_{0}(0), \quad v(+\infty)=0 .
$$

Again there exists an exponentially decreasing solution $v_{0}$. In this case it can be expressed by means of cylinder functions. This fact indicates that a numerical scheme for solving (61) which converges uniformly with respect to $\varepsilon$ should reflect the complicated boundary structure, especially near $x=0$.

Similarly to the investigations in Section 2 we denote by $\bar{p}$ and $\bar{f}$ a piecewise constant approximation of the given functions $p$ and $f$, respectively. As in the original problem we assume $\bar{p}(x) \geq p_{0}>0$ in $(0,1)$ with some constant $p_{0}$. 
Now, an approximate solution $u_{h}$ of the singularly perturbed boundary value problem (61) is defined by

$$
-\varepsilon^{2} \frac{d}{d x}\left(x^{\alpha} \frac{d u_{h}}{d x}\right)+\bar{p}(x) u_{h}=\bar{f}(x), \quad x \in(0,1), \quad u_{h}(0)=u_{h}(1)=0 .
$$

This problem has to be interpreted in the weak form as in the unperturbed case, i.e. we need to find some $u_{h} \in H_{0}^{1, \alpha}(\Omega)$ such that

$$
\begin{aligned}
\varepsilon^{2} \int_{\Omega} x^{\alpha} u_{h}^{\prime}(x) v^{\prime}(x) d x+\int_{\Omega} \bar{p}(x) u_{h}(x) v(x) d x= & \int_{\Omega} \bar{f}(x) v(x) d x \\
& \text { for any } v \in H_{0}^{1, \alpha}(\Omega) .
\end{aligned}
$$

For any fixed $\varepsilon \neq 0$ and for any $h>0$ problem (64) has a unique solution $u_{h}$. Furthermore, the following error equation holds:

$$
-\varepsilon^{2} \frac{d}{d x}\left(x^{\alpha} \frac{d\left(u_{h}-u\right)}{d x}\right)+\bar{p}(x)\left(u_{h}-u\right)=\bar{f}-f+(p-\bar{p}) u \quad \text { a.e. in }(0,1)
$$

and the boundary conditions

$$
\left(u_{h}-u\right)(0)=\left(u_{h}-u\right)(1)=0
$$

are satisfied. Using the bound $\|u\|_{L^{\infty}} \leq c$ which is independent of the perturbation parameter $\varepsilon \neq 0$, from (65), (66) we obtain

THEOREM 7. Let $\bar{p}$ and $\bar{f}$ denote some first order piecewise constant approximations of the functions $p$ and $f$ respectively. Then there is some constant $c$ independent of $\varepsilon \neq 0$ such that

$$
\left\|u-u_{h}\right\|_{L^{\infty}} \leq c h
$$

If, additionally, the conditions $f \geq 0, \bar{p} \leq p, \bar{f} \geq f$ are satisfied then we have $u_{h}(x) \geq u(x)$ for any $x \in[0,1]$.

Finally, we give some numerical results obtained in the example

$$
-\varepsilon^{2} \frac{d}{d x}\left(x^{\alpha} \frac{d u}{d x}\right)+\left(1+x^{2}\right) u=2+\sin (2 \pi x), \quad x \in(0,1), \quad u(0)=u(1)=0 .
$$

For the finite-dimensional realization we again use the representation of the solution $u_{h}$ of (63) in the form

$$
u_{h}(x)=\sum_{i=1}^{N-1} u_{i} \varphi_{i}(x)+\sum_{i=1}^{N} w_{i} \psi_{i}(x) .
$$

In the present case the adapted base functions $\varphi_{i}$ and $\psi_{i}$ are supposed to satisfy

$$
-\varepsilon^{2} \frac{d}{d x}\left(x^{\alpha} \frac{d \varphi_{i}}{d x}\right)+\bar{p} \varphi_{i}=0, \quad \varphi_{i}\left(x_{j}\right)=\delta_{i j}
$$

and

$$
-\varepsilon^{2} \frac{d}{d x}\left(x^{\alpha} \frac{d \psi_{i}}{d x}\right)+\bar{p} \psi_{i}=1, \quad \varphi_{i}\left(x_{j}\right)=0
$$


respectively. Similarly to [42] the base functions defined by (69), (70) are higher transcendental functions, in our case cylinder functions. As for the unperturbed problems the smoothness of the approximate solution $u_{h}$ results in conditions at the inner grid points $x_{i}$. From the representation (68) we obtain a tridiagonal linear system, i.e. the unknown coefficients $u_{i}$ are determined by

$$
a_{i} u_{i-1}+b_{i} u_{i}+c_{i} u_{i+1}=r_{i}, \quad i=1(1) N-1,
$$

with $u_{0}=u_{N}=0$ and the coefficients

$$
a_{i}=\varphi_{i-1}^{\prime}\left(x_{i}-0\right), \quad b_{i}=\varphi_{i}^{\prime}\left(x_{i}-0\right)-\varphi_{i}\left(x_{i}+0\right), \quad c_{i}=-\varphi_{i+1}^{\prime}\left(x_{i}+0\right)
$$

and the right hand side

$$
r_{i}=-f_{i} \psi_{i}^{\prime}\left(x_{i}-0\right)+f_{i+1} \psi_{i+1}^{\prime}\left(x_{i}+0\right) .
$$

Now, we discuss the system (71). From the maximum principle we obtain $\varphi_{i}(x) \geq$ 0 . Further, the defining equation (69) results in

$$
-\left.\varepsilon^{2} x^{\alpha} \frac{d \varphi_{i}}{d x} \varphi_{i}\right|_{x_{i-1}} ^{x_{i}}+\varepsilon^{2} \int_{x_{i-1}}^{x_{i}} x^{\alpha}\left(\frac{d \varphi_{i}}{d x}\right)^{2} d x+\int_{x_{i-1}}^{x_{i}} \bar{p}\left(\varphi_{i}\right)^{2} d x=0 .
$$

Thus, $\varphi_{i}^{\prime}\left(x_{i}-0\right)>0$ holds. In a similar way one can show that $a_{i}<0, b_{i}>0$, $c_{i}<0$. Together with the regularity of system (71), which is a consequence of the coercivity of the operator related to (63), this proves the matrix corresponding to (71) to be an M-matrix.

An essential problem in applying the proposed method consists in the determination of the functions $\varphi_{i}, \psi_{i}$ which satisfy (69), (70). The base functions can be represented by

$$
\varphi_{i}(x)= \begin{cases}k_{i 1} v_{i 1}(x)+k_{i 2} v_{i 2}(x) & \text { for } x \in\left[x_{i-1}, x_{i}\right], \\ d_{i 1} v_{i 1}(x)+d_{i 2} v_{i 2}(x) & \text { for } x \in\left[x_{i}, x_{i+1}\right], \\ 0 & \text { otherwise }\end{cases}
$$

with appropriate coefficients $k_{i 1}, k_{i 2}, d_{i 1}, d_{i 2}$ such that $\varphi_{i}\left(x_{j}\right)=\delta_{i j}$. The functions $v_{i 1}, v_{i 2}$ are defined by

$$
\begin{aligned}
& v_{i 1}(x)=x^{(1-\alpha) / 2} I_{(1-\alpha) /(2-\alpha)}\left(\frac{2 \sqrt{p_{i}}}{\varepsilon(2-\alpha)} x^{(2-\alpha) / 2}\right), \\
& v_{i 2}(x)=x^{(1-\alpha) / 2} K_{(1-\alpha) /(2-\alpha)}\left(\frac{2 \sqrt{p_{i}}}{\varepsilon(2-\alpha)} x^{(2-\alpha) / 2}\right) .
\end{aligned}
$$

Here $I_{\nu}$ and $K_{\nu}$ denote the modified Bessel functions of the first kind and of the second kind, respectively, of order $\nu$. Furthermore, the functions $\psi_{i}$ are given by

$$
\psi_{i}(x)= \begin{cases}\left(1 / p_{i}\right)\left(1-\varphi_{i-1}(x)-\varphi_{i}(x)\right) & \text { for any } x \in\left[x_{i-1}, x_{i}\right] \\ 0 & \text { otherwise }\end{cases}
$$

In [1] truncated Taylor expansions have been considered to approximate the required base functions $\varphi_{i}, \psi_{i}$. However, this approach does not guarantee a uniform (w.r.t. $\varepsilon$ ) convergence of the approximate scheme. 
In our numerical experiments we applied an iterative technique with a simplified operator to generate the solution $u_{h}$ of the auxiliary problem (63). Let us define mappings $b(\cdot, \cdot), c(\cdot, \cdot): H_{0}^{1, \alpha}(\Omega) \times H_{0}^{1, \alpha}(\Omega) \rightarrow \mathbb{R}$ by

$$
\begin{aligned}
& b(u, v)=\varepsilon^{2} \int_{\Omega} x^{\alpha} u^{\prime}(x) v^{\prime}(x) d x+\sum_{i=1}^{N-1} d_{i} u_{i} v_{i}, \\
& c(u, v)=\sum_{i=1}^{N-1} d_{i} u_{i} v_{i}+\int_{\Omega} \bar{f}(x) v(x) d x-\int_{\Omega} \bar{p}(x) u(x) v(x) d x .
\end{aligned}
$$

Here $d_{i}=\left(h_{i} p_{i}+h_{i+1} p_{i+1}\right) / 2$ and $p_{i}, p_{i+1}$ denote the values of the function $\bar{p}$ in the intervals $\Omega_{i}$ and $\Omega_{i+1}$, respectively. The sequence $\left\{u^{k}\right\} \subset H_{0}^{1, \alpha}(\Omega)$ is defined via

$$
b\left(u^{k+1}, v\right)=c\left(u^{k}, v\right) \quad \text { for any } v \in H_{0}^{1, \alpha}(\Omega) .
$$

This is equivalent to

$$
-\varepsilon^{2} \frac{d}{d x}\left(x^{\alpha} \frac{d u^{k+1}}{d x}\right)=\bar{f}-\bar{p} u^{k}, \quad x \neq x_{i},
$$

and

$$
\begin{aligned}
\varepsilon^{2} x_{i}^{\alpha}\left(\left(u^{k+1}\right)^{\prime}\left(x_{i}-0\right)-\left(u^{k+1}\right)^{\prime}\left(x_{i}+0\right)\right)+d_{i} u_{i}^{k+1}=d_{i} u_{i}^{k}, & \\
i & =1(1) N-1 .
\end{aligned}
$$

We use the function $u^{0} \equiv 0$ as starting iterate in (76), (77). Let functions $\zeta_{j}, \eta_{j}$ on $\bar{\Omega}$ be defined by

$$
\zeta_{j}(x)=x^{j(2-\alpha)}, \quad j=0,1, \ldots, \quad \text { and } \quad \eta_{j}(x)=x^{j(2-\alpha)-1}, \quad j=1,2, \ldots
$$

These functions satisfy

$$
-\left(x^{\alpha} \zeta_{j}^{\prime}\right)^{\prime}=\varrho_{j} \zeta_{j-1}, \quad j=1,2, \ldots, \quad \text { and } \quad-\left(x^{\alpha} \eta_{j}^{\prime}\right)^{\prime}=\sigma_{j} \eta_{j-1}, \quad j=2,3, \ldots,
$$

with

$$
\begin{aligned}
& \varrho_{j}=-j(2-\alpha)[j(2-\alpha)-1+\alpha], \quad j=1,2, \ldots, \quad \text { and } \\
& \sigma_{j}=-(j-1)(2-\alpha)[j(2-\alpha)-1], \quad j=2,3, \ldots
\end{aligned}
$$

Furthermore, we have

$$
-\left(x^{\alpha} \zeta_{0}^{\prime}\right)^{\prime}=0 \quad \text { and } \quad-\left(x^{\alpha} \eta_{1}^{\prime}\right)^{\prime}=0
$$

Because $\bar{f}, \bar{p}$ are piecewise constant the functions $u^{k}$ generated by (76), (77) can be represented locally in the subintervals by

$$
u^{k}(x)=\sum_{j=0}^{k} s_{i j}^{k} \zeta_{j}(x)+\sum_{j=1}^{k} t_{i j}^{k} \eta_{j}(x), \quad x \in \Omega_{i},
$$

with coefficients $s_{i j}^{k}, t_{i j}^{k} \in \mathbb{R}$. The piecewise differential equations (76) result in

$$
s_{i, j}^{k+1}=-\frac{p_{i}}{\varepsilon^{2} \varrho_{j}} s_{i j-1}^{k}, \quad t_{i, j}^{k+1}=-\frac{p_{i}}{\varepsilon^{2} \sigma_{j}} t_{i j-1}^{k}, \quad j=2(1) k, i=1(1) N,
$$


and

$$
s_{i 1}^{k+1}=\frac{1}{\varepsilon^{2}}\left[f_{i}-\frac{p_{i}}{\varrho_{1}} s_{i 0}^{k}\right], \quad i=1(1) N .
$$

The remaining coefficients $s_{i 0}^{k+1}, t_{i 1}^{k+1}$ are determined by the differentiability, i.e. from $u^{k+1} \in C^{1}(\bar{\Omega})$, and by the boundary conditions $u^{k+1}(0)=u^{k+1}(1)=0$ and by the equations (77). As in the previous cases this can be reduced to tridiagonal linear systems provided appropriate representations are used.

The sequence generated by (76), (77) converges to the required solution $u_{h}$ of the auxiliary problem (63) only if the step size $h>0$ is small enough. This can be avoided by solving (63) with the exact bases $\varphi_{i}, \psi_{i}$ given by (69), (70).

In the following table we present the achieved accuracies $\delta_{h}:=\max _{0 \leq i \leq N}\left\{\bar{u}_{i}-\right.$ $\left.\underline{u}_{i}\right\}$ for different values $\varepsilon^{2}$ and various step sizes on equidistributed grids.

\begin{tabular}{|c|cccc|c|}
\hline \multirow{2}{*}{$N \backslash \varepsilon^{2}$} & \multicolumn{5}{|c|}{$\alpha=0.5$} \\
\cline { 2 - 6 } & 0.1 & 0.01 & 0.001 & 0.0001 & $\alpha=0.8$ \\
\hline 50 & $7.055 \mathrm{E}-2$ & $1.104 \mathrm{E}-1$ & $1.257 \mathrm{E}-1$ & - & $1.129 \mathrm{E}-1$ \\
100 & $3.533 \mathrm{E}-2$ & $5.530 \mathrm{E}-2$ & $6.305 \mathrm{E}-2$ & - & $5.648 \mathrm{E}-2$ \\
200 & $1.767 \mathrm{E}-2$ & $2.766 \mathrm{E}-2$ & $3.154 \mathrm{E}-2$ & $3.209 \mathrm{E}-2$ & $2.825 \mathrm{E}-2$ \\
\hline
\end{tabular}

The sign "_" in the table indicates that the iteration method used failed. In these cases Bessel functions have to be used to represent the solution. The results given above show a good agreement with Theorem 7 stating an $\varepsilon$-uniform linear convergence of the proposed method. The maximal difference between the generated upper and lower solutions varies mildly only if the parameters $\varepsilon$ or $\alpha$ are changed.

6. Other problems and techniques. In this section we briefly refer to other problems which can be handled by monotone discretization as well as to some other techniques for generating enclosures in differential equations.

The monotone discretization takes advantage of some monotonicity behaviour of the differential operator. These properties occur in several further classes of problems as shown in [51]. Thus, the idea of monotone discretization could be applied to a wider range of problems.

In [32], [39] the method was used to generate two-sided bounds of the solution of 1D-parabolic problems. By means of Rothe's method of semi-discretization in time the original problem was transformed into a sequence of two-point boundary value problems which can be handled by the technique described. To guarantee a pointwise enclosure by Rothe's method the right hand side of the differential equation was properly shifted.

In [13] the monotone discretization method was applied to systems of twopoint boundary value problems.

Initial value problems for ordinary differential equations form another field of possible applications of monotone discretization. However, the wrapping effect 
blows up the generated bounds if no special treatment is used. Here a combination with the method proposed in [36] could be useful.

A serious obstacle for the trivial extension of monotone discretization to partial differential equations consists in the fact that the generated auxiliary problems have to be solved analytically. This restricts the method to specific problems and to special classes of differential operators. Especially in the case of elliptic and of higher-dimensional parabolic problems the monotone discretization concept has to be modified. First attempts in this direction can be found in [18], [50].

Finally, let us briefly mention two other approaches for generating two-sided enclosures of the solution of differential equations. In [4], [6], [7], for example, a powerful technique of reliable numerical algorithms has been investigated. The basic principle of these algorithms consists in a Taylor expansion of the solution combined with an appropriate shift. Further specific implementations using interval arithmetics make these methods applicable to a large range of problems. A special method for high accuracy bounds of the solution of initial value problems was introduced in [36].

Local linearizations and adapted monotone modifications of iteration methods form the base of a new type of methods proposed in e.g. [38], [46], [47]. These methods can also be used to handle nonmonotone branches of nonlinear problems. Furthermore, the underlying fixed point principles enable one to derive existence results. Thus, these algorithms form a constructive tool for solving nonlinear problems.

\section{References}

[1] H. Abhari and C. Grossmann, Approximate bases for boundary value problems and applications to MID-techniques, Z. Angew. Math. Mech. 72 (1992), 83-91.

[2] H. Abhari and M. Al-Zanaidi, Computational aspects of monotone iteration discretization algorithm, preprint, TU Dresden, 07-01-88.

[3] R. A. Adams, Sobolev Spaces, Academic Press, New York 1975.

[4] E. Adams, Invers-Monotonie, direkte und indirekte Intervallmethoden, Forschungszentrum Graz, Ber. 185 (1982)

[5] E. Adams, R. Ansorge, C. Grossmann and H.-G. Roos (eds.), Discretization in Differential Equations and Enclosures, Akademie-Verlag, Berlin 1987.

[6] E. Adams, D. Cordes and H. Keppler, Enclosure methods as applied to linear periodic ODEs and matrices, Z. Angew. Math. Mech. 70 (1990), 565-578.

[7] E. Adams und H. Spreuer, Konvergente numerische Schrankenkonstruktionen mit Spline-Funktionen für nichtlineare gewöhnliche bzw. parabolische Randwertaufgaben, in: K. Nickel (ed.), Interval Mathematics, Springer, Berlin 1975.

[8] M. Al-Zanaidi and C. Grossmann, Monotone discretization in boundary value problems using PASCAL-SC, in: I. Marek (ed.), Proc. ISNA 87, Teubner, Leipzig 1988, 91-96.

[9] —, - Monotone iteration discretization algorithm for BVP's, Computing 31 (1989), 5974.

[10] - - , 1D-grid generation by monotone iteration discretization, ibid. 34 (1990), 377-390.

[11] N. Anderson and A. M. Arthurs, Variational solutions of the Thomas-Fermi equations, Quart. Appl. Math. 39 (1981), 127-129. 
[12] S. Carl, The monotone iterative technique for a parabolic boundary value problem with discontinuous nonlinearity, Nonlinear Anal. 13 (1989), 1399-1407.

[13] S. Carl and C. Grossmann, Iterative spline bounds for systems of boundary value problems, in: J. W. Schmidt and H. Späth (eds.), Splines in Numerical Analysis, AkademieVerlag, Berlin 1989, 19-30.

[14] - - - Monotone enclosure for elliptic and parabolic systems with nonmonotone nonlinearities, J. Math. Anal. Appl. 151 (1990), 190-202.

[15] S. Carl and S. Heikkilä, On extremal solutions of an elliptic boundary value problem involving discontinuous nonlinearities, preprint, Univ. Oulu, 1990; to appear in Differential Integral Equations.

[16] C. Y. Chan and Y. C. Hon, A constructive solution for a generalized Thomas-Fermi theory of ionized atoms, Quart. Appl. Math. 45 (1987), 591-599.

[17] R. C. Duggan and C. Goodmann, Pointwise bounds for a nonlinear heat conduction model of the human head, Bull. Math. Biol. 48 (1986), 229-236.

[18] A. Felgenhauer, Monotone discretization of the Poisson equation in the plane, to appear.

[19] R. C. Flagg, C. D. Luning and W. L. Perry, Implementation of new iterative techniques for solutions of Thomas-Fermi and Emden-Fowler equations, J. Comput. Phys. 38 (1980), 396-405.

[20] E. C. Gartland, On the uniform convergence of the Scharfetter-Gummel discretization, preprint, Kent State Univ., Feb. 1991.

[21] E. C. Gartland and C. Grossmann, Semianalytic solution of boundary value problems by using approximated operators, Z. Angew. Math. Mech. 72 (1992), 615-619.

[22] C. Grossmann, Monotone Einschließung höherer Ordnung für 2-Punkt-Randwertaufgaben, Z. Angew. Math. Mech. 67 (1987), T475-T477.

[23] - Monotone discretization of two-point boundary value problems and related numerical methods, in [5], 99-122.

[24] - Semianalytic discretization of weakly nonlinear boundary value problems with variable coefficients. Z. Anal. Anwendungen 10 (1991), 513-523.

[25] - Enclosures of the solution of the Thomas-Fermi equation by monotone discretization, J. Comput. Phys. (1992), 26-38.

[26] C. Grossmann und M. Krätzschmar, Monotone Diskretisierung und adaptive Gittergenerierung für Zwei-Punkt-Randwertaufgaben, Z. Angew. Math. Mech. 65 (1985), T264T266.

[27] C. Grossmann, M. Krätzschmar und H.-G. Roos, Gleichmäßig einschließende Diskretisierungsverfahren für schwach nichtlineare Randwertaufgaben, Numer. Math. 49 (1986), 95-110.

[28] C. Grossmann and H.-G. Roos, Feedback grid generation via monotone discretization for two-point boundary-value problems, IMA J. Numer. Anal. 6 (1986), 421-432.

[29] - - - Uniform enclosure of high order for boundary value problems by monotone discretization, Math. Comp. 53 (1989), 609-617.

[30] - - - Convergence analysis of higher order monotone discretization, Wiss. Z. Tech. Univ. Dresden 38 (1989), 155-168.

[31] —, - Enclosing discretization for singular and singularly perturbed boundary value problems, in: H.-G. Roos, A. Felgenhauer and L. Angermann (eds.), Numerical Methods in Singular Perturbed Problems, TU Dresden, 1991, 71-82.

[32] G. Koeppe, H.-G. Roos and L. Tobiska, An enclosure generating modification of the method of discretization in time, Comment. Math. Univ. Carolin. 28 (1987), 447-453.

[33] M. Krätzschmar, Iterationsverfahren zur Lösung schwach nichtlinearer elliptischer Randwertaufgaben mit monotoner Lösungseinschließung, Dissertation, TU Dresden, 1983. 
[34] U. Kulisch (ed.), PASCAL-SC, Pascal Extension for Scientific Computation, Wiley, New York and Teubner, Stuttgart 1987.

[35] G. S. Ladde, V. Lakshmikantham and A. S. Vatsala, Monotone Iterative Techniques for Nonlinear Differential Equations, Pitman, 1985.

[36] R. Lohner, Einschließung der Lösung gewöhnlicher Anfangs- und Randwertaufgaben und Anwendungen, Dissertation, Univ. Karlsruhe, 1988.

[37] J. M. Ortega and W. C. Rheinboldt, Iterative Solution of Nonlinear Equations in Several Variables, Academic Press, New York 1970.

[38] M. Plum, Numerical existence proofs and explicit bounds for solutions of nonlinear elliptic boundary value problems, to appear.

[39] H.-G. Roos, The Rothe method and monotone discretization for parabolic equations, in [5], 155-166.

[40] - Uniformly enclosing discretization methods and grid generation for semilinear boundary value problems with first order terms, Apl. Mat. 34 (1989), 274-284.

[41] _- A uniformly convergent discretization method for singularly perturbed boundary value problems of the fourth order, Zb. Rad. Prirod.-Mat. Fak. Novi Sad Ser. Mat. 19 (1989), $51-64$.

[42] - Global uniformly convergent schemes for a singularly perturbed boundary-value problem using patched base spline functions, J. Comput. Appl. Math. 29 (1990), 69-77.

[43] - Uniform enclosing discretization methods for semilinear boundary value problems, in: Nonlinear Analysis and Mathematical Modelling, Banach Center Publ. 24, PWN Warszawa 1990, 257-268.

[44] J. W. Schmidt, Ein Einschließungsverfahren für Lösungen fehlerbehafteter linearer Gleichungen, Period. Math. Hungar. 13 (1982), 29-37.

[45] J. Schröder, Operator Inequalities, Academic Press, New York 1980.

[46] —, A method for producing verified results for two-point boundary value problems, in: Comput. Suppl. 6, Springer, Vienna 1988, 9-22.

[47] -, Operator inequalities and applications, in: E. W. Norrie (ed.), Inequalities, Marcel Dekker, New York 1991, 163-210.

[48] J. Sprekels and H. Voss, Pointwise inclusions of fixed points by finite dimensional iteration schemes, Numer. Math. 32 (1979), 381-392.

[49] G. Vanden Berghe and H. De Meyer, Accurate computation of higher Sturm-Liouville eigenvalues, to appear.

[50] R. Voller, Enclosure of solutions of weakly nonlinear elliptic boundary value problems and their computation, Computing 42 (1989), 245-258.

[51] W. Walter, Differential and Integral Inequalities, Springer, Berlin 1970.

[52] J. Weissinger, A kind of difference methods for enclosing solutions of ordinary linear boundary value problems, in: Comput. Suppl. 6, Springer, Vienna 1988, 23-32. 